\title{
Recovery from rat sciatic nerve injury in vivo through the use of differentiated MDSCs in vitro
}

\author{
XIANGYI ZENG ${ }^{1}$, LI ZHANG $^{2}$, LIANG SUN $^{2}$, DAI ZHANG $^{3}$, HENGWU ZHAO $^{1}$, \\ JUN JIA $^{1}$ and WEI WANG ${ }^{2}$ \\ ${ }^{1}$ The Third Affiliated Hospital of Liaoning Medical University; ${ }^{2}$ Jinzhou Clinical Institute of Liaoning Medical \\ University/Jinzhou Central Hospital; ${ }^{3}$ Liaoning Medical University, Jinzhou, Liaoning 121000, P.R. China
}

Received April 23, 2012; Accepted September 18, 2012

DOI: $10.3892 /$ etm.2012.785

\begin{abstract}
In this study, muscle-derived stem cells (MDSCs) whose differentiation into neuron-like cells was induced by ciliary neurotrophic factor (CNTF) and Salvia (Salvia miltiorrhiza) in vitro were used to repair rat sciatic nerve injuries in vivo, in order to investigate their multifunctional characteristics as pluripotent stem cells. The sciatic nerve in the right side of the lower limb was exposed under the anesthetized condition of $10 \%$ chloral hydrate $(0.3 \mathrm{ml} / 100 \mathrm{~g})$ injection into the abdominal cavity. The tissue which was $0.5 \mathrm{~cm}$ above the sciatic nerve bifurcation was broken using a hemostat. After induction, MDSCs were transferred in sodium hyaluronate gel and were placed into the damaged area. An untreated control group was also included in this study. The surgical area was sutured after washing with gentamycin sulfate solution. Sciatic nerve function index (SFI) was calculated, electrophysiological tests were performed and the recovery rate of gastrocnemius muscle wet weight was also calculated. Four weeks post-surgery, the SFI and the recovery rate of gastrocnemius muscle wet weight in the MDSC group were significantly higher than those in the control group $(\mathrm{P}<0.05)$. MDSCs whose differentiation is induced by CNTF and Salvia play an active role in the repair of peripheral nerve injury.
\end{abstract}

\section{Introduction}

Limb joint and soft tissue injuries are among the most common conditions in clinical practice and are often followed by peripheral nerve injury in 5\% of cases. The peripheral nerve is a mixed nerve, and nerve fibers emanating from one side cross over to the other within nerve bundles. Therefore, the complete recovery rate is only $10-25 \%$ after injury and treatment of peripheral nerve injuries remains a challenge worldwide.

Correspondence to: Dr Wei Wang, Second section 51\#, Shanghai Road, Guta, Jinzhou, Liaoning 121000, P.R. China

E-mail:weiwang_ly@yahoo.com.cn

Key words: muscle-derived stem cell, epineurium, sciatic nerve function index, recovery rate of gastrocnemius muscle wet weight
Several researchers have performed numerous studies in this field and proposed that the basic condition for peripheral nerve repair is the construction of the nerve regenerative chamber, which can create a specific microenvironment for regeneration of the nerve $(1,2)$. As stem cell research has developed, study into novel tissue engineering seed cells has received increasing attention. Muscle-derived stem cells (MDSCs) originate from muscle tissue. In addition to aspects shared by stem cells, MDSCs also exhibit such characteristics as harmlessness to the body, a wide range of sources, ready acceptance by patients, lack of restrictions of source of donor, lack of immunosuppressive effects and fewer ethical restrictions. MDSCs are therefore ideal seed cells for peripheral nerve repair (3-6). As the study of Chinese traditional medicine has developed, it has been confirmed that Salvia (Salvia miltiorrhiza) may be used to induce the differentiation of multi-functional stem cells into neuron-like cells (7-10).

In the present study, MDSCs whose differentiation was induced by ciliary neurotrophic factor (CNTF) and Salvia were used to repair sciatic nerve injury in rats in order to further confirm their potential as pluripotent stem cells. This study may contribute to a theoretical concept of more latent cytokines and novel seed cells for the construction of tissue engineered peripheral nerve grafts.

\section{Materials and methods}

Experimental animals. Adult Sprague-Dawley (SD) rats $(n=12)$ were divided into 2 groups. The sciatic nerve in the right lower limb was exposed under the anesthetized condition of $10 \%$ chloral hydrate $(0.3 \mathrm{ml} / 100 \mathrm{~g})$ injection into the abdominal cavity. The tissue, which was $0.5 \mathrm{~cm}$ above the sciatic nerve bifurcation, was broken using a hemostat. After induction, MDSCs were transferred to sodium hyaluronate gel and placed into the damaged area. An untreated control group was also included in this study. The surgical area was sutured after washing with gentamycin sulfate solution. Animal experiments were performed in accordance with the Guide for the Care and Use of Laboratory Animals.

\section{Experimental materials}

General observation. The recovery of the wound and the formation of ulcers in the plantar region were recorded. Under 
Table I. Experimental data in the MDSC and control group.

\begin{tabular}{lcccc}
\hline Group & SFI & $\begin{array}{c}\text { Action } \\
\text { potential }\end{array}$ & $\begin{array}{c}\text { Nerve } \\
\text { conductive velocity }\end{array}$ & $\begin{array}{c}\text { Recovery rate of } \\
\text { gastrocnemius muscle wet weight }(\%)\end{array}$ \\
\hline MDSC & $-87.11 \pm 0.88^{\mathrm{a}}$ & $13.58 \pm 0.53^{\mathrm{a}}$ & $23.72 \pm 0.62^{\mathrm{a}}$ & $57.6 \pm 0.0094^{\mathrm{a}}$ \\
Control & $-88.54 \pm 0.64$ & $12.79 \pm 0.69$ & $22.99 \pm 0.61$ & $56.59 \pm 0.0076$ \\
\hline
\end{tabular}

${ }^{\mathrm{a}} \mathrm{P}<0.05$. SFI, sciatic nerve function index.

mild anesthesia, the sensory function recovery was examined following plantar puncture.

Sciatic nerve function index (SFI). Four weeks after surgery, SFI was calculated using the method described by Reynolds and Weiss (11). Hind legs of the rats were dyed with ink. When the rats walked on the surface of one piece of white paper, the footprints of healthy feet $(\mathrm{N})$ and wounded feet $(\mathrm{E})$ were measured in 3 indices as follows: length of footprint (IPL, from toe to heel), width of toes (ITS, from the 1st to the 5 th toe) and width of middle toes (IIT, from the 2 nd to the 4 th toe). The results should be accurate to $0.1 \mathrm{~mm}$. SFI was calculated according to the formula described by Bain et al (12): $\mathrm{SFI}=-38.3[(\mathrm{EPL}-\mathrm{NPL}) / \mathrm{NPL}]+109.5[(\mathrm{ETS}-\mathrm{NTS}) / \mathrm{NTS}]$ $+13.3[(\mathrm{EIT}-\mathrm{NIT}) / \mathrm{NIT}]-8.8$. A SFI value between 0 and $11 \%$ represented normal nerve function, whereas $-100 \%$ represented complete damage of nerve function, and -11 to $-100 \%$ incomplete nerve function recovery.

Electrophysiology test. Four weeks after surgery, the sciatic nerve at the surgical site was exposed under anesthesia. The transplanted section was linked with an electrode and the gastrocnemius was linked with a recording electrode to determine the action potential (AP) and mean conductive velocity (MCV) of the sciatic nerve.

Recovery rate of gastrocnemius wet weight. Four weeks following surgery, the gastrocnemius was completely removed and its wet weight was then measured by ER-182 electronic balance $(1 / 2,000 \mathrm{~g})$ to calculate its recovery rate.

Statistical analysis. All statistic data are presented as the means \pm SD. statistical analyses were performed using SPSS 10.0 software. A P-value $<0.05$ was considered to indicate a significant difference and $\mathrm{P}<0.01$ a statistically significant difference.

\section{Results}

General observation. Following surgery it was found that the toes of the rats were crooked and muscle contraction was not observed when the toes were punctured. One week after surgery, it was found that the surgical area had recovered well without infection. However, there was redness and swelling on the skin of the right foot and weight-bearing area. All the rats were inactive and the operated legs were immobile. Two weeks after surgery, ulcers were observed on the skin behind the ankle of the operated area in some rats, while other rats exhibited redness and swelling on the soles of their feet. There were no obvious differences between the MDSC and control groups.

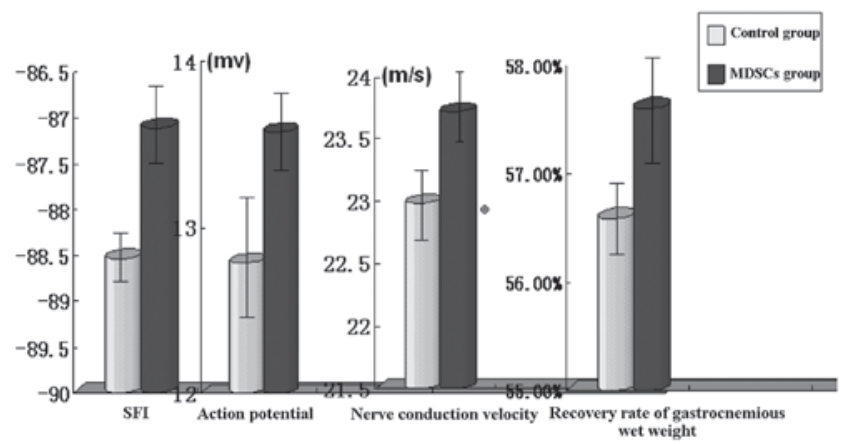

Figure 1. Sciatic nerve function index (SFI), action potential, nerve conductive velocity and recovery rate of gastrocnemius wet weight in the MDSC and control groups.

Three weeks after surgery, the rats' moods had recovered and the treated legs became active. However, the toes and knee joint remained crooked with various degrees of muscle shrinkage in the treated legs. At the same time, inflammation and ulcers were found in the plantar region. Four weeks after surgery, the knee joints of all the rats remained crooked; however, the inflamed area and ulcers in the plantar region were no longer observed. All the rats reacted when their toes were punctured. In the MDSC group, there were 3 rats that generally recovered from inflammation and ulcers, and partly recovered from muscle shrinkage. However, all the rats in the control group exhibited various degrees of muscle shrinkage in the treated legs.

SFI. SFI was statistically higher in the MDSC group in comparison with the control group $(\mathrm{P}<0.05$; Table I and Fig. 1).

Electrophysiology test. Sciatic action potential and nerve conductive velocity were statistically higher in the MDSC group in comparison with the control group $(\mathrm{P}<0.05$; Table I and Fig. 1).

Recovery rate of gastrocnemius wet weight. The recovery rate of gastrocnemius wet weight was statistically higher in the MDSC group in comparison with the control group $(\mathrm{P}<0.05$; Table I and Fig. 1).

\section{Discussion}

In Chinese traditional medicine, peripheral nerve injury is considered to belong to the category of flaccidity. Flaccidity is a syndrome with such symptoms as weak tendons and feeble 
muscle, leading to muscle shrinkage due to lack of bodily exercise.

Nerve damage can cause blood stasis and meridian obstruction, leading to poor circulation and malnutrition. Bodily functions therefore exhibit anomalous changes and pathological changes occur in such a situation.

Modern experimental research has confirmed that medicine promoting blood circulation to remove blood stasis can achieve favorable results in the treatment of disease caused by peripheral nerve injury. Qian et al (13) demonstrated that Bu Yang Huan Wu Tang reduces the shrinkage of neurons in injured peripheral nerve and aids nerve recovery. Fang et al (14) confirmed that such compound Chinese herbal medicine as ginseng, Astragalus and Salvia can promote peripheral nerve regeneration.

As a result of technical developments in genetic engineering, cells with biological activity are used as carriers for nutritional nerve factor. After modification, the carrier cells can constantly provide nutritional nerve factor to the gene, which provides a prospective application for nerve restoration and regeneration with nutritional nerve factor and which also gives hope to nerve tissue engineering of cell types.

MDSCs originate from muscle. They are precursor cells of skeletal muscle cells. Compared with other cells, they exhibit such characteristics as partial differentiation ability, favorable histocompatibility, harmlessness to the body, wide range of sources and ready acceptance by patients. Several investigators have noted that MDSCs originating from skeletal muscle have the characteristics of stem cells whose differentiation is induced by CNTF (15-26). This study used the liquid culture method allowing the generation of a large number of MDSCs and the induction of MDSCs to obtain amplification ability. MDSCs after the 3rd generation may be used for cell differentiation and cell treatment. As seed cells, MDSCs play a substantial role in the restoration of rat sciatic nerve injury and regeneration of the peripheral nerve under the effect of Salvia.

Three elements of nerve tissue engineering include seed cells, nerve carrier and nerve nutritional factor. The source and large proliferation of seed cells are the most important problems to be solved. In addition, other research on bionic scaffold material has received increasing attention. Research on nerve carriers has also made substantial progress.

Hyaluronic acid is a linear polysaccharide macromolecular material, which comprises $(1 \rightarrow 4)$-D-glucuronic acid- $\beta$ $(1 \rightarrow 3)-\mathrm{N}$-acetyl glucosamine disaccharide units and is widely present in the connective tissue of humans and animals. With a 3-dimensional network structure formed by interaction between molecules, it has such characteristics as maintaining tissue formation, important psychological function and good viscoelasticity. Glucuronic acid is also a main element in compounding cellular and extracellular matrices, which occupy vital roles in maintaining normal physiological function, supporting the growth and differentiation of cells and restoration of injured tissue. In the early period of wounding, such cells as fibroblast cells and macrophages exhibit a marked increase in compounding glucuronic acid in the function of growth factor. It has been shown in the comparison between the embryo before and after birth that glucuronic acid maintains high thickness, which prevents collagenous fibrous tissue from contracting and even aids tissue recovery without any cicatricial sign. After birth, glucuronic acid remains at high thickness in the early period; later it is gradually dissolved by hyaluronidase. At the same time, the combination of collagenous fiber increases. Finally, cicatricial restoration is formed. Additionally, glucuronic acid has such functions as promoting blood vessel growth, partly improving circulation of injured areas and aiding growth factor expression. There are high quantities of carboxyl and hydroxyl in glucuronic acid molecules. These two elements can form an intermolecular and intramolecular hydrogen bond in water with such potency that it can hold 1,000 times its weight in water $(27,28)$.

In the present study, sodium hyaluronate gel was used as a cell carrier. In the recovery process, the extracellular matrix had an effect on cell performance. Cells also modified the extracellular matrix in various ways, forming the micro-environment. The whole recovery process, which was constantly changing, supported the growth of the cells. In conclusion, the restoration and regeneration of tissue is a process in which cells composed of different molecules and macromolecules proliferate, differentiate and reconstruct the matrix.

\section{References}

1. Mackinnon SE, Dellon AL, Hudson AR and Hunter DA: Nerve regeneration through a pseudosynovial sheath in a primate model. Plast Reconstr Surg 75: 833-841, 1985.

2. Seckel BR: Enhancement of peripheral nerve regeneration. Muscle Nerve 13: 785-800, 1990.

3. Wallace GQ, Lapidos KA, Kenik JS and McNally EM: Long-term survival of transplanted stem cells in immunocompetent mice with muscular dystrophy. Am J Pathol 173: 792-802, 2008.

4. Shimazaki T, Shingo T and Weiss S: The ciliary neurotrophic factor/leukemia inhibitory factor/gp130 receptor complex operates in the maintenance of mammalian forebrain neural stem cells. J Neurosci 21: 7642-7653, 2001.

5. Helgren ME, Squinto SP, Davis HL, Parry DJ, Boulton TG, Heck CS, Zhu Y, Yancopoulos GD, Lindsay RM and DiStefano PS: Trophic effect of ciliary neurotrophic factor on denervated skeletal muscle. Cell 11: 493-504, 1994.

6. Rivera FJ, Kandasamy M, Couillard-Despres S, Caioni M, Sanchez R, Huber C, Weidner N, Bogdahn U and Aigner L: Oligodendrogenesis of adult neural progenitors: differential effects of ciliary neurotrophic factor and mesenchymal stem cell derived factors. J Neurochem 107: 832-843, 2008.

7. Xia W, Xiang P, Zhang L, Chen Z, Yu W, Zhang X, Li Y and Li S: Human mesenchymal stem cells differentiate into neuronlike cells with Tanshinone II A. Zongguo Bing Li Sheng Li Za Zhi 19: 865-869, 2003 (In Chinese).

8. Ma L, Feng X, Yang L, Xie Q, Luo M and Huang T: Induction of human umbilical cord blood mesenchymal stem cells into nerve-like cells by Salvia miltiorrhiza. Shiyong Erke Linchuang Zazhi 20: 1125-1128, 2005 (In Chinese).

9. Sanchez-Ramos J, Song S, Cardozo-Pelaez F, et al: Adult bone marrow stromal cells differentiate into neural cells in vitro. Exp Neurol 164: 247-256, 2000.

10. Yu Q, Lian J and Guo Y: Experimental study on differentiation of bone mesenchymal stem cells into neuron-like cells with Salvia miltiorrhiza injection. Zhongguo Zhong Xi Yi Jie He Ji Jiu Za Zhi 13: 210-213, 2006

11. Reynolds BA and Weiss S: Generation of neurons and astrocytes from isolated cells of the adult mammalian central nervous system. Science 255: 1707-1710, 1992.

12. Bain JR, Mackinnon SE and Hunter DA: Functional evaluation of complete sciatic, peroneal, and posterior tibial nerve lesions in the rat. Plast Reconstr Surg 83: 129-138, 1989

13. Qian Y, Tao Y, Huang C and Wu Y: Experimental study of the survival-promoting effect of Buyang Huanwu decoction on neurons after peripheral nerve injury. Zhonghua Shou Waike Zazhi 9: 152-154, 2002 (In Chinese).

14. Fang Y, Chen D and Gu Y: Facilitated regeneration of rat peripheral nerves with mixture of dangshen, astragalus root, red sage root. Zhonghua Shou Waike Zazhi 14: 181-183, 1998 (In Chinese). 
15. Zeng X, Wang W, Sun L, Zhang L and Zeng L: Differentiation of muscle-derived stem cells into neuron-like cells induced by ciliary neurotrophic factor and compound Salvia miltiorrhiza injection in vitro. Zhonghua Zu Zhi Gong Cheng Yan Jiu 13: 5336-5340, 2009 (In Chinese).

16. Blanco-Bose WE, Yao CC, Kramer RH and Blau HM: Purification of mouse primary myoblast based on alpha 7 integrin expression. Exp Cell Res 265: 212-220, 2001.

17. Galli R, Borello U, Gritti A, et al: Skeletal myogenic potential of human and mouse neural stem cells. Nat Neurosci 3: 986-991, 2000.

18. Kuznetsov SA, Mankani MH, Gronthos S, et al: Circulating skeletal stem cells. J Cell Biol 153: 1133-1140, 2001.

19. Chen S, Zhang Q, Wu X, et al: Dedifferentiation of lineagecommitted cells by a small molecule. J Am Chem Soc 126 410-411, 2004

20. Orellana RA, Suryawan A, Kimball SR, Wu G, Nguyen HV, Jefferson LS and Davis TA: Insulin signaling in skeletal muscle and liver of neonatal pigs during endotoxemia. Pediatr Res 64: $505-510,2008$

21. Chen X, Mao Z, Liu S, et al: Dedifferentiation of adult human myoblasts induced by ciliary neurotrophic factor in vitro. Mol Biol Cell 16: 3140-3151, 2005.
22. Zhu W, Shiojima I, Ito Y, et al: IGFBP-4 is an inhibitor of canonical Wnt signalling required for cardiogenesis. Nature 454: 345-349, 2008.

23. Cebolla B and Vallejo M: Nuclear factor-I regulates glial fibrillary acidic protein gene expression in astrocytes differentiated from cortical precursor cells. J Neurochem 97: 1057-1070, 2006.

24. Bhattacharya I and Ullrich A: Endothelin-1 inhibits adipogenesis: role of phosphorylation of Akt and ERK1/2. FEBS Lett 580: 5765-5771, 2006

25. Xie Y, Yu Z, Liu L, Guo Y and Lou L: Biphasic regulation of extracellular signal-regulated kinases by scalaradial, a secretory phospholipase $\mathrm{A}(2)$ inhibitor. Cancer Biol Ther 5: 988-992, 2006.

26. Mukherjee A and Rotwein P: Insulin-like growth factor-binding protein-5 inhibits osteoblast differentiation and skeletal growth by blocking insulin-like growth factor actions. Mol Endocrinol 22: 1238-1250, 2008

27. Morrison SJ, Shah NM and Anderson DJ: Regulatory mechanisms in stem cell biology. Cell 88: 287-298, 1997.

28. Lundberg C, Martínez-Serrano A, Cattaneo E, et al: Survival, integration, and differentiation of neural stem cell lines after transplantation to the adult rat striatum. Exp Neurol 145: 342-360, 1997. 